\title{
Clinical Study \\ Pulmonary Hypertension in Portugal: First Data from a Nationwide Registry
}

\author{
Rui Baptista, ${ }^{1,2}$ José Meireles, ${ }^{3}$ Ana Agapito, ${ }^{4}$ Graça Castro, ${ }^{1}$ \\ António Marinho da Silva, ${ }^{1}$ Teresa Shiang, ${ }^{5}$ Fabienne Gonçalves, ${ }^{3}$ Susana Robalo-Martins, ${ }^{6}$ \\ António Nunes-Diogo, ${ }^{6}$ and Abílio Reis ${ }^{3}$ \\ ${ }^{1}$ Department of Cardiology, Hospitais da Universidade de Coimbra, Centro Hospitalar e Universitário de Coimbra, \\ Praceta Mota Pinto, 3000 Coimbra, Portugal \\ ${ }^{2}$ Institute for Biomedical Imaging and Life Sciences, Faculty of Medicine of University of Coimbra, \\ Azinhaga de Santa Comba, 3000 Coimbra, Portugal \\ ${ }^{3}$ Department of Internal Medicine, Hospital Geral de Santo António, Centro Hospitalar do Porto, \\ Largo Professor Abel Salazar, 4099 Porto, Portugal \\ ${ }^{4}$ Department of Cardiology, Hospital Santa Marta, Centro Hospitalar de Lisboa Central, Rua de Santa Marta, 1169 Lisboa, Portugal \\ ${ }^{5}$ Department of Pneumology, Hospital Santos Silva, Centro Hospitalar Gaia/Espinho, Rua Conceição Fernandes, \\ 4434 Vila Nova de Gaia, Portugal \\ ${ }^{6}$ Department of Cardiology, Hospital de Santa Maria, Centro Hospitalar de Lisboa Norte, Avenida Professor Egas Moniz, \\ 1649 Lisboa, Portugal
}

Correspondence should be addressed to Rui Baptista; rui.baptista@fmed.uc.pt

Received 8 April 2013; Accepted 26 August 2013

Academic Editor: Zhirong Sun

Copyright (c) 2013 Rui Baptista et al. This is an open access article distributed under the Creative Commons Attribution License, which permits unrestricted use, distribution, and reproduction in any medium, provided the original work is properly cited.

\begin{abstract}
Introduction. Pulmonary arterial hypertension $(\mathrm{PAH})$ is a rare disease that must be managed in specialized centers; therefore, the availability of epidemiological national data is critical. Methods. We conducted a prospective, observational, and multicenter registry with a joint collaboration from five centers from Portugal and included adult incident patients with PAH or chronic thromboembolic pulmonary hypertension (CTEPH). Results. Of the 79 patients enrolled in this study, 46 (58.2\%) were classified as PAH and 33 patients (41.8\%) as CTEPH. PAH patients had a mean age of $43.4 \pm 16.4$ years. Idiopathic PAH was the most common etiology (37\%). At presentation, PAH patients had elevated right atrial pressure (RAP) $(7.7 \pm 5.9 \mathrm{mmHg})$ and mean pulmonary vascular resistance $\left(11.4 \pm 6.5\right.$ Wood units), with a low cardiac index $\left(2.7 \pm 1.1 \mathrm{~L} \cdot \mathrm{min}^{-1} \cdot \mathrm{m}^{-2}\right)$; no patient was under selective pulmonary vasodilators; however, at follow-up, most patients were on single (50\%), double (28\%), or triple (9\%) combination vasodilator therapy. One-year survival was $93.5 \%$, similar to CTEPH patients (93.9\%), that were older (60.0 \pm 12.5 years) and had higher RAP $(11.0 \pm 5.2 \mathrm{mmHg}, P=0.015)$. Conclusions. We describe for the first time nationwide data on the diagnosis, management, and prognosis of PAH and CTEPH patients in Portugal. Clinical presentation and outcomes are comparable with those reported on other national registries.
\end{abstract}

\section{Introduction}

In the past few decades, the international scientific community has made great progresses in the understanding of the epidemiology, pathophysiology, and management of pulmonary arterial hypertension (PAH). It is a rare disease, malignant in character, and rapidly fatal, if not treated, with a median survival of 2.8 years in a historic cohort [1].
These progresses were accompanied by the development of drugs that target specific pathways in the pathophysiology of the disease [2]. Management in specialized centers and the use of pulmonary vasodilators lead to a significant impact on the survival and quality of life of PAH patients [3]. Unfortunately, survival rates are still unsatisfactory [4], signaling for the need of more effective treatments, which are under development [5]. 
Since the first consensus conference in 1973 [6], the classification of pulmonary hypertension $(\mathrm{PH})$ has evolved, reflecting the ongoing understanding of the condition, and now it includes five groups with several subtypes [7]. Within group $1 \mathrm{PH}$, an idiopathic subgroup is maintained, highlighting that there is still a lot to understand about the pathogenesis of the disease. The diagnostic and therapeutic approach should be guided by national and international guidelines supported by scientific societies, and given the rarity and severity of the disease, its proper investigation and treatment should be performed in expert centers $[8,9]$.

Chronic thromboembolic pulmonary hypertension (CTEPH), classified as group $4 \mathrm{PH}$, has a different pathophysiology and treatment from other $\mathrm{PH}$ groups. Pulmonary endarterectomy (PEA) is a potentially curative procedure for CTEPH [10]. For those patients not eligible for surgery or those with persistent $\mathrm{PH}$ after PEA, specific treatment may ameliorate symptoms and enhance survival $[9,11]$.

The organization and publication of national and international registries are essential in the understanding of the epidemiology, etiology, and natural history of the different groups of PH [3]. Several groups have published data from their cohorts [12-17], although with different inclusion and exclusion criteria and methodologies [16]; to overcome these disparities, the creation of an international registry has been suggested [18]. Moreover, it is unclear if data from regional registries can be applied to other populations [13]. Data from national registries are not a surrogate for application in other countries and cannot be easily extrapolated due to demography, treatment availability, and other regional differences. Therefore, national registries from each region are paramount in the interpretation of the applicability of international recommendations, which are issued regardless of those differences. Our aim is to present data from a Portuguese registry of patients with group 1 and group $4 \mathrm{PH}$ and to compare them with other published cohorts.

\section{Population and Methods}

We conducted a prospective, observational, and multicenter registry with a joint collaboration from five $\mathrm{PH}$ centers around Portugal. Although there are small differences between the institutions regarding patient follow-up, all of them follow similar protocols, according to the published national [8] and international [19] guidelines. Our study population consisted of adult incident $\mathrm{PH}$ patients referred to those centers for diagnostic and therapeutic evaluation, between 2008 and 2010. Data were collected by clinical file review by a physician, with supervision from the assistant $\mathrm{PH}$ physician, and were compiled in a dedicated software, specifically developed for the management of $\mathrm{PH}$ patients (PAHTool, Inovultus, Santa Maria da Feira, Portugal), creating a database and the backbone for a national registry. An informed consent was obtained from each patient, and the study protocol conforms to the ethical guidelines of the 1975 Declaration of Helsinki as reflected in a priori approval by the institution's human research committee. The centers' participation in this registry was voluntary, and the nationwide data collection was approved by the National Center for Data Protection.

To enable comparisons with other published registries, we used strict inclusion and exclusion criteria. All patients had PAH confirmed by right heart catheterization (RHC), with a mean pulmonary arterial pressure (PAP) over $25 \mathrm{mmHg}$ and a pulmonary wedge pressure (PCWP) equal or under $15 \mathrm{mmHg}$ or a left ventricular end diastolic pressure (LVEDP) equal or under $15 \mathrm{mmHg}$. The date of diagnosis corresponds to the confirmation of PAH by RHC.

Studied data included demographic characteristics, clinical and laboratorial parameters, World Health Organization (WHO) functional class, haemodynamics, and conventional and specific vasodilator therapy usage and survival status. Vasoreactivity testing was performed when possible, using various institutional protocols. A one-year follow-up was conducted; no patients were lost to follow-up.

All results are expressed as the mean \pm standard deviation or as the frequency. We used Kolmogorov-Smirnov for testing normality, Student's $t$-test for continuous variables, and $X^{2}$ test for categorical variables. Survival analysis was performed using the Kaplan-Meier method, and comparisons were made using the Log-Rank test. Values of $P<0.05$ were considered to be significant. Statistical analysis was performed using SPSS 17.0 software package (IBM, New York, USA).

\section{Results}

Our registry originally included $188 \mathrm{PH}$ patients (Figure 1). After exclusion of 79 patients from groups 2, 3, and $5 \mathrm{PH}, 134$ patients were left for analysis. Thirty patients were excluded as they did not have an available RHC. The final analysis included 79 patients. Of the 79 patients enrolled in this study, $46(58.2 \%)$ patients were classified as $\mathrm{PAH}$ and 33 patients (41.8\%) as CTEPH.

3.1. Demographics and Clinical Data. There was a clear preponderance of women among PAH patients, with a female/ male patient ratio of $1.9: 1$. Mean age at diagnosis was $43.4 \pm$ 16.4 years (range, 15 to 77 years) (Table 1). There was no difference among genders regarding age at first medical examination $(P=0.963)$. Among the 46 patients, $9.2 \%(n=$ $11)$ were $<21$ years old, $58.3 \%(n=21)$ were 21 to 40 years old, $32.6 \%(n=15)$ were 40 to 59 years old, and $17.4 \%(n=8)$ were $>61$ years old. Patients between 21 and 60 years of age accounted for $87 \%$ of all patients.

Idiopathic PAH was present in 17 (37\%) patients, followed by connective tissue disease (CTD) $(n=12,26 \%)$, congenital heart disease (CHD) $(n=10,22 \%)$, portopulmonary hypertension $(n=5,11 \%)$, familial $(n=1,2 \%)$, and other etiologies $(n=1,2 \%)$ (Table 2). At baseline, most patients presented in WHO class III or IV (71\%); only one patient was in class I.

CTEPH patients had a higher mean age at diagnosis $(60.3 \pm 12.5, P<0.001)$ than group $1 \mathrm{PAH}$ patients; a significant proportion of the population had more than 51 years at diagnosis (63.6\%) (Figure 2). Both WHO class at presentation and the female/male ratio were similar to group $1 \mathrm{PAH}$ patients. 


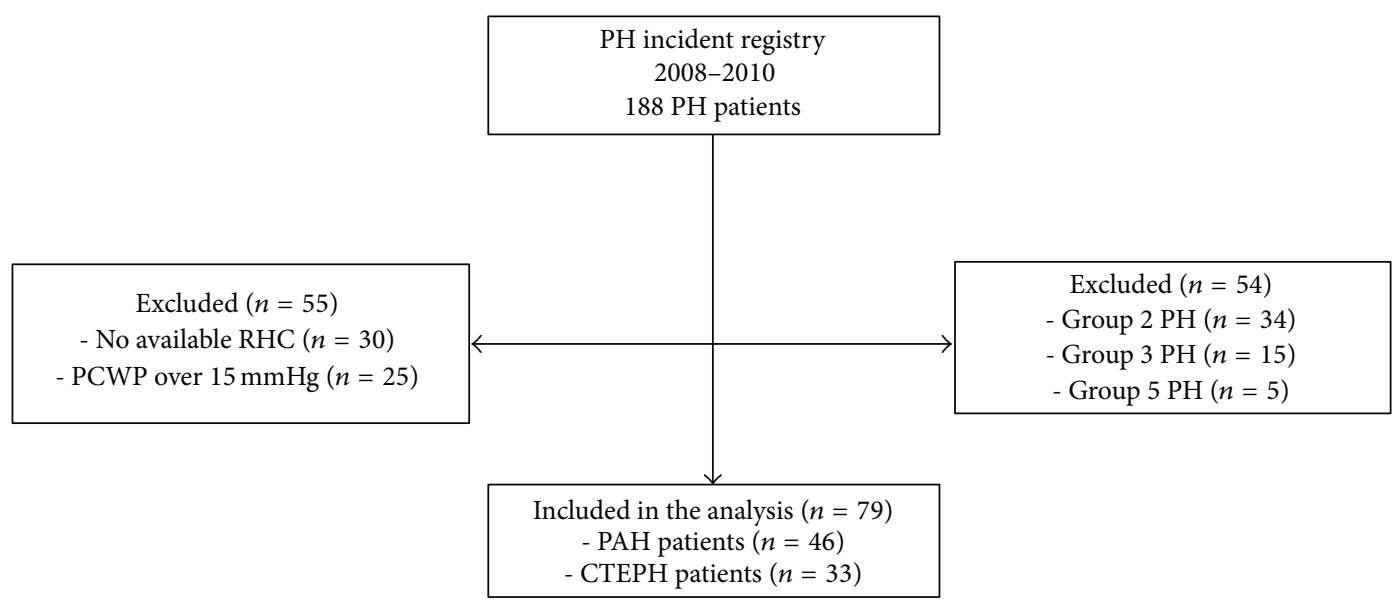

Figure 1: Patient selection flowchart.

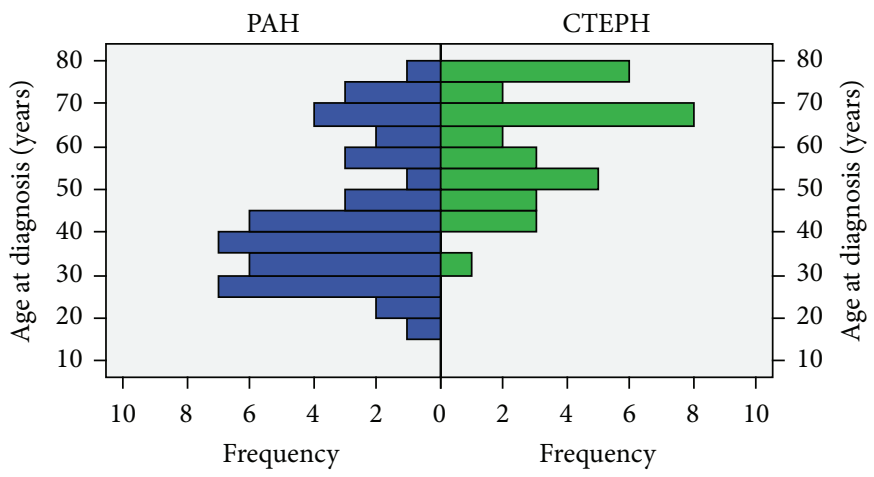

Figure 2: Distribution of age and gender.

3.2. Hemodynamics. RHC was performed in all patients at the initial examination (Table 1). Baseline data shows that in group $1 \mathrm{PAH}$ patients, mean RAP was $7.7 \pm 5.9 \mathrm{mmHg}$, mean PAP was $50.6 \pm 17.9 \mathrm{mmHg}$, and mean PCWP was $9.5 \pm 3.5 \mathrm{mmHg}$; PVR was $11.4 \pm 6.5$ Wood units. Mean cardiac output $(\mathrm{CO})$ was $4.5 \pm 1.8 \mathrm{~L} \cdot \mathrm{min}^{-1}$, and mean cardiac index $(\mathrm{CI})$ was $2.7 \pm 1.1 \mathrm{~L} \cdot \mathrm{min}^{-1} \cdot \mathrm{m}^{-2}$. Cardiac output was more elevated in WHO class I/II than in the WHO class III or IV patients, but it did not reach statistical significance. Conversely, PVR was higher in patients in WHO class III/IV than patients in WHO class I/II (Table 3). Vasoreactivity testing was performed in $29(63.0 \%)$ patients with various protocols; 6 patients $(21 \%)$ had a positive test.

Regarding CTEPH, the only hemodynamic parameter at the time of diagnostic RHC that was significantly different from PAH was the mean RAP $(11.0 \pm 5.2 \mathrm{mmHg}, P=0.015)$, that was significantly higher.

3.3. Treatment. Drug therapy at study inclusion is shown in Table 4. At baseline, all PAH patients were treated only with conventional therapy. Diuretics were used by 15 patients (32.6\%), followed by oxygen in 9 patients $(19.6 \%)$ and digoxin in 7 patients (15.2\%). At follow-up, 42 patients were treated with advanced PAH therapies and 40 with pulmonary vasodilators, and two patients were enrolled in randomized controlled trials (RCT) (Table 5). Most patients were medicated with endothelin receptor antagonists $(n=$ $33)$, followed by phosphodiesterase inhibitors $(n=26)$ and prostanoids $(n=4)$. Thirteen patients $(28 \%)$ were under double combination therapy and 4 (9\%) patients under triple combination therapy.

No differences were found regarding baseline treatment modalities among PAH and CTEPH patients. However, during follow-up, targeted therapies were begun in $67 \%$ of CTEPH patients, and 5 patients (15.2\%) had a PEA. Combination therapy was offered to $9 \mathrm{CTEPH}$ patients during the follow-up period. Endothelin receptor antagonists were used in 17 patients, followed by sildenafil in 13 patients and prostanoids in 2 patients. One patient was enrolled in a RCT.

3.4. One-Year Survival Analysis. Survival data was available for all patients (Figure 3). One year after the diagnostic RHC, 5 patients were deceased. The Kaplan-Meier survival estimates for patients with PAH and CTEPH at 1 year were $93.5 \%$ and $93.9 \%$, respectively (Log-rank $P=0.709$ ). Unoperated CTEPH patients had a one-year survival rate of $92.9 \%$, whereas all patients that underwent PEA survived.

3.5. Comparison with the Cohort of Group 1 PAH Patients without Available Baseline RHC. The original database included 
TABLE 1: Demographic, clinical, and hemodynamic characteristics of pulmonary arterial hypertension (PAH) and chronic thromboembolic pulmonary hypertension (CTEPH) incident patients at baseline.

\begin{tabular}{|c|c|c|c|c|}
\hline & Total $(n=79)$ & $\mathrm{PAH}(n=46)$ & CTEPH $(n=33)$ & $P$ value \\
\hline Age (years) & $50.5 \pm 17.0$ & $43.4 \pm 16.4$ & $60.3 \pm 12.5$ & $<0.001$ \\
\hline Female gender, $n(\%)$ & $53(67.1 \%)$ & $30(65.2 \%)$ & $23(69.7 \%)$ & 0.676 \\
\hline Six-minute test walking distance $(\mathrm{m})$ & $351.3 \pm 137.4$ & $370.8 \pm 140.1$ & $320.4 \pm 132.9$ & 0.327 \\
\hline \multicolumn{5}{|l|}{ Functional class, $n(\%)$} \\
\hline I & $1(1 \%)$ & $1(2 \%)$ & $0(0 \%)$ & 0.565 \\
\hline II & $17(25 \%)$ & $11(27 \%)$ & $6(21 \%)$ & \\
\hline III & $34(49 \%)$ & $21(51 \%)$ & $13(46 \%)$ & \\
\hline IV & $17(25 \%)$ & $8(20 \%)$ & $9(32 \%)$ & \\
\hline \multicolumn{5}{|l|}{ Hemodynamic data } \\
\hline Right atrial pressure $(\mathrm{mmHg})$ & $9.1 \pm 5.8$ & $7.7 \pm 5.9$ & $11.0 \pm 5.2$ & 0.015 \\
\hline Mean pulmonary artery pressure $(\mathrm{mmHg})$ & $49.1 \pm 15.1$ & $50.6 \pm 17.9$ & $47.1 \pm 9.9$ & 0.313 \\
\hline Cardiac output $\left(\mathrm{L} \cdot \mathrm{min}^{-1}\right)$ & $4.4 \pm 1.9$ & $4.5 \pm 1.8$ & $4.3 \pm 2$ & 0.778 \\
\hline Cardiac index $\left(\mathrm{L} \cdot \mathrm{min}^{-1} \cdot \mathrm{m}^{-2}\right)$ & $2.6 \pm 1.1$ & $2.7 \pm 1.1$ & $2.5 \pm 1.1$ & 0.406 \\
\hline Pulmonary capillary wedge pressure (mmHg) & $9.7 \pm 3.3$ & $9.5 \pm 3.5$ & $9.9 \pm 3.1$ & 0.587 \\
\hline Pulmonary vascular resistance (Wood units) & $11.1 \pm 6.4$ & $11.4 \pm 6.5$ & $10.8 \pm 6.3$ & 0.729 \\
\hline
\end{tabular}

TABLE 2: Clinical and hemodynamic data stratified by pulmonary arterial hypertension subgroup.

\begin{tabular}{lcccccccccc}
\hline Subgroup & $N(\%)$ & Female (\%) & $\begin{array}{c}\text { Age } \\
\text { (years) }\end{array}$ & $\begin{array}{c}\text { WHO I/II } \\
(\%)\end{array}$ & $\begin{array}{c}\text { 6MWT } \\
(\text { meters })\end{array}$ & $\begin{array}{c}\text { RAP } \\
(\mathrm{mmHg})\end{array}$ & $\begin{array}{c}\text { mPAP } \\
(\mathrm{mmHg})\end{array}$ & $\begin{array}{c}\text { CO } \\
\left(\mathrm{L} \cdot \mathrm{min}^{-1}\right)\end{array}$ & $\begin{array}{c}\text { PCW } \\
(\mathrm{mmHg})\end{array}$ & $\begin{array}{c}\text { PVR } \\
(\mathrm{WU})\end{array}$ \\
\hline Idiopathic & $17(37.0)$ & 70.6 & $37.5 \pm 12.9$ & 31.3 & $405 \pm 121$ & $11 \pm 6$ & $53 \pm 15$ & $4.2 \pm 1.5$ & $10.7 \pm 3.3$ & $11.7 \pm 5.6$ \\
CTD & $12(26.1)$ & 75.0 & $56.8 \pm 12.4$ & 27.3 & $275 \pm 127$ & $6 \pm 6$ & $39 \pm 11$ & $4.9 \pm 1.8$ & $7.6 \pm 3.2$ & $8.7 \pm 7$ \\
CHD & $10(21.7)$ & 50.0 & $37.7 \pm 15$ & 22.2 & $351 \pm 171$ & $6 \pm 5$ & $60 \pm 27$ & $4.6 \pm 2.7$ & $10.7 \pm 3.7$ & $13.9 \pm 8.9$ \\
PortPulm & $5(10.9)$ & 60.0 & $51.2 \pm 18.3$ & 33.3 & $\mathrm{n} / \mathrm{d}$ & $7 \pm 5$ & $51 \pm 11$ & $4 \pm 1.2$ & $8.6 \pm 2.9$ & $11.1 \pm 3.3$ \\
\hline Total & $\mathbf{4 4}(\mathbf{1 0 0 . 0})^{*}$ & 65.2 & $43.4 \pm 16.4$ & 29.3 & $371 \pm 140$ & $8 \pm 6$ & $51 \pm 18$ & $4.5 \pm 1.8$ & $9.5 \pm 3.5$ & $11.4 \pm 6.5$ \\
\hline
\end{tabular}

CTD: connective tissue disease; CHD: congenital heart disease; PortPulm: portopulmonary. WHO: World Health Organization; 6MWT: six-minute walking test distance; RAP: right atrial pressure; mPAPA: mean pulmonary artery pressure; CO: cardiac output; PCWP: pulmonary capillary wedge pressure; PVR: pulmonary vascular resistance; WU: wood units.

${ }^{*}$ Heritable PAH $(n=1)$ and other etiologies PAH $(n=1)$ were not reported as there was one case of each in the cohort.

TABLE 3: Hemodynamic characteristics stratified by NYHA class of pulmonary arterial hypertension incident patients.

\begin{tabular}{|c|c|c|c|c|}
\hline$(n=46)$ & NYHA I/II & NYHA III & NYHA IV & $P$ value \\
\hline Six-minute walk test distance $(\mathrm{m})$ & $436 \pm 147$ & $356 \pm 106$ & $236 \pm 128$ & 0.094 \\
\hline Female gender & $67.8 \%$ & $71.4 \%$ & $37.5 \%$ & 0.229 \\
\hline Right atrial pressure $(\mathrm{mmHg})$ & $8 \pm 6$ & $8 \pm 6$ & $7 \pm 7$ & 0.854 \\
\hline Mean pulmonary artery pressure $(\mathrm{mmHg})$ & $54 \pm 26$ & $49 \pm 11$ & $45 \pm 13$ & 0.492 \\
\hline Pulmonary capillary wedge pressure $(\mathrm{mmHg})$ & $9 \pm 3$ & $10 \pm 4$ & $7 \pm 3$ & 0.092 \\
\hline Cardiac output $\left(\mathrm{L} \cdot \mathrm{min}^{-1}\right)$ & $5.4 \pm 0.9$ & $4.6 \pm 2.1$ & $3.2 \pm 1.6$ & 0.097 \\
\hline Pulmonary vascular resistance (Wood units) & $9.1 \pm 4.6$ & $10.6 \pm 5.6$ & $15.3 \pm 6.1$ & 0.123 \\
\hline
\end{tabular}

TABLE 4: Conventional therapies at baseline and follow-up of pulmonary arterial hypertension (PAH) and chronic thromboembolic pulmonary hypertension $(\mathrm{CTEPH})$ patients.

\begin{tabular}{lcccccc}
\hline & \multicolumn{2}{c}{ Total } & \multicolumn{2}{c}{ PAH } & \multicolumn{2}{c}{ CTEPH } \\
& Baseline & Follow-up & Baseline & Follow-up & Baseline & Follow-up \\
\hline Diuretics & $33(41.8 \%)$ & $40(65.6 \%)$ & $15(32.6 \%)$ & $17(60.7 \%)$ & $18(54.5 \%)$ & $23(69.7 \%)$ \\
Digoxin & $13(16.5 \%)$ & $18(2.8 \%)$ & $7(15.2 \%)$ & $11(23.9 \%)$ & $6(21.2 \%)$ \\
Oxygen & $21(26.6 \%)$ & $25(31.6 \%)$ & $9(19.6 \%)$ & $12(26.1 \%)$ & $12(36.4 \%)$ & $13(39.4 \%)$ \\
Warfarin & $34(43.0 \%)$ & $59(74.7 \%)^{*}$ & $10(21.7 \%)$ & $28(60.9 \%)^{*}$ & $24(72.7 \%)$ & $31(93.9 \%)^{*}$ \\
\hline
\end{tabular}

${ }^{*} P<0.001$ versus baseline. 


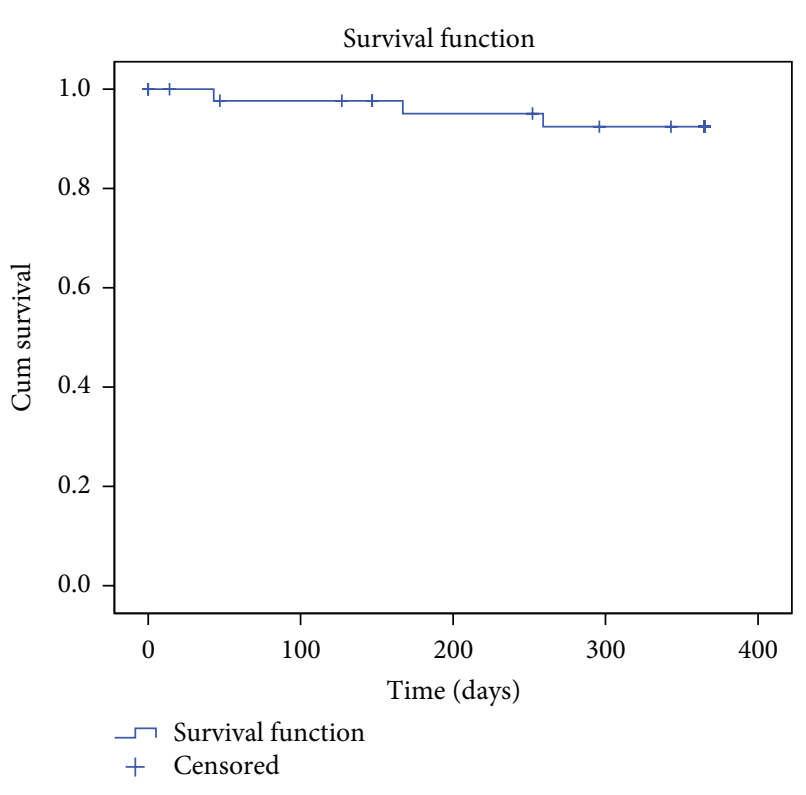

(a)

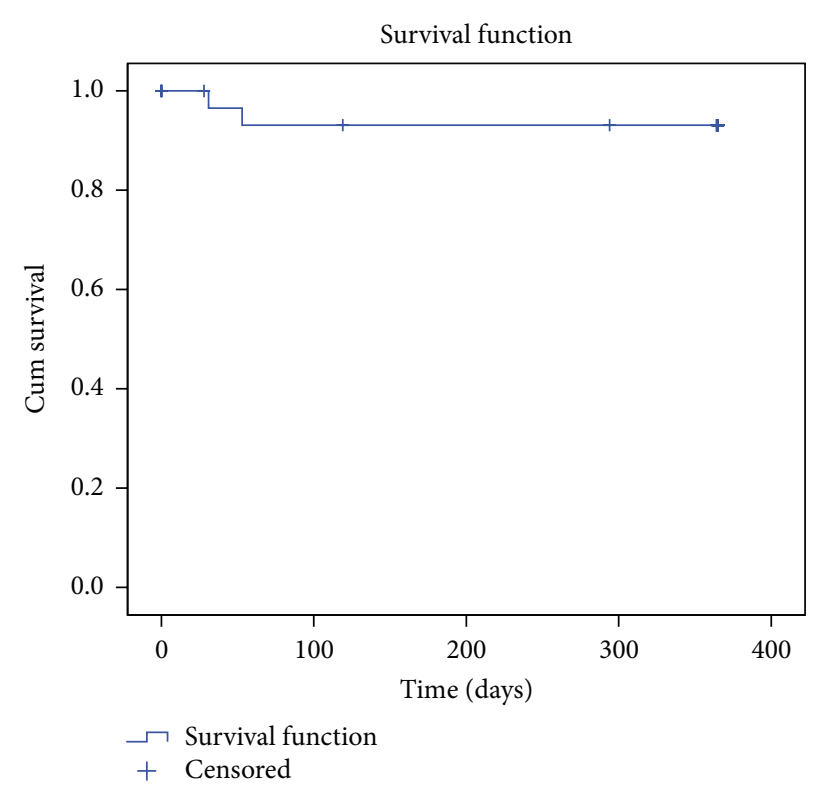

(b)

FIGURE 3: One-year survival in pulmonary arterial hypertension (a) and chronic thromboembolic pulmonary hypertension (b) patients.

TABLE 5: Pulmonary vasodilator therapies at follow-up of pulmonary arterial hypertension (PAH) and chronic thromboembolic pulmonary hypertension (CTEPH) patients.

\begin{tabular}{lcc}
\hline & PAH & CTEPH \\
\hline No advanced therapies & $2 \%$ & $33 \%$ \\
Single therapy & $50 \%$ & $36 \%$ \\
Double combination therapy & $28 \%$ & $15 \%$ \\
Triple combination therapy & $9 \%$ & $7 \%$ \\
Calcium channel blockers & $7 \%$ & $0 \%$ \\
Randomized controlled trial drug & $4 \%$ & $3 \%$ \\
\hline
\end{tabular}

20 patients classified as PAH but without an available baseline RHC; therefore, they were not included in the analysis. This may have been due to the incomplete filling of the database fields and thus not necessarily reflecting the absence of RHC. Comparing with included $\mathrm{PAH}$ patients, we found no significant differences regarding gender, age, or WHO class at presentation of these patients. Although not reaching statistical significance, there was a trend for a higher proportion of patients with CHD-associated PAH in the group of patients that did not had a RHC. No survival differences were found among the two groups.

3.6. Estimated Incidence of PAH and CTEPH. Although limited by the voluntary collection of data and by the selective inclusion and exclusion criteria, we identified 46 patients with incident group $1 \mathrm{PAH}$ and 33 patients with CTEPH during the 3-year follow-up period. For a population of 10 million inhabitants in Portugal, we calculated a conservative estimation of group land group $4 \mathrm{PH}$ annual incidence of at least 1.5 and 1.1 patients per million, respectively. However, if we include patients with a clinical diagnosis of group $1 \mathrm{PAH}$ but without an available RHC, our incidence would rise to 2.2 per million per year.

\section{Discussion}

The present study summarizes data representative of the Portuguese $\mathrm{PH}$ cohort. With the combined effort of five treatment centers, we were able for the first time to collect nationwide data on the diagnosis, management, and clinical course of PAH and CTEPH in Portugal.

To analyze a homogeneous population and to enable comparisons with other published cohorts $[12,17,20]$, we followed strict inclusion and exclusion criteria based on current guidelines [9]. We focused only on PAH and CTEPH patients, as the prevalence and clinical characteristics of group 2 and group $3 \mathrm{PH}$ varied widely among the five $\mathrm{PH}$ centers. Additionally, we included only incident cases to remove survivor bias from our study and to permit an approximate calculation of annual incidence, as prevalent cases correspond mainly to survivors [12].

In our PAH population, age at diagnosis was lower than in the REVEAL [20] and French [12] cohorts but higher than in the NIH registry [21]. This may be due to the fact that one-fifth of our patients had $\mathrm{CHD}$-associated $\mathrm{PAH}$; these patients were excluded from the French registry [12] but not from the REVEAL cohort [20]. Other important aspect is that almost $20 \%$ of $\mathrm{PAH}$ patients were over 60 years, a finding that is being increasingly recognized in contemporary registries. CTEPH patients were older, with a mean age at diagnosis of 60 years, similar to the one found in the Swiss cohort [22] and the randomized clinical trial [23]. Interestingly, our CTEPH population was significantly older than a published cohort of patients developing CTEPH after an acute pulmonary embolism [24]. This fact may warrant further investigation 
and may signalize a different epidemiology of postpulmonary embolism CTEPH.

Possibly due to the fact that we have no patients with PAH secondary to anorexigens, a group almost exclusively formed by women [25], the proportion of female patients (65\%) in our PAH cohort was lower than that in most published reports [26].

Idiopathic $\mathrm{PAH}$ was the most frequent subgroup of $\mathrm{PAH}$, with a proportion of $37 \%$, similar to the French cohort (41\% in the incident cohort) [12] but lower than in the REVEAL cohort (47\%) [20]. CTD-PAH was the second most common cause, with $26 \%$, a number that is higher than that reported on the French (18\%) [12] but similar to the REVEAL cohort (24\%) [20]. Systemic sclerosis is the leading cause of CTD-PAH, with $8 \%$ of patients developing this dismal prognostic finding in the course of their disease [27]; echocardiographic screening may be of value and has a grade IIb C recommendation on the current ESC guidelines [9].

As expected, patients with CHD-PAH were frequent in our series (22\%), a significantly higher proportion than in the French and REVEAL cohorts $[12,20]$. This may be the result of the poor access of CHD patients to corrective heart surgery in the appropriate age; however, CHD patients are also clearly underrepresented in other epidemiological series, as in the French cohort due to health organization issues [12]. Portopulmonary hypertension had a similar incidence to the French series.

PAH baseline haemodynamics was similar to those from the NIH, REVEAL French Comparison Cohort (FCC), and French registry. The mean PAP was $51 \mathrm{mmHg}$, being essentially the same of the French registry $(55 \mathrm{mmHg})$ [12] and the REVEAL FCC $(51 \mathrm{mmHg})$ [26] and slightly lower than the NIH cohort (60 $\mathrm{mmHg}$ ) [1]. Mean RAP at diagnosis was $8 \mathrm{mmHg}$, the same as the French $(8 \mathrm{mmHg})$ and REVEAL FCC registry $(8 \mathrm{mmHg})$.

Most PAH patients presented to the referral center with symptoms of advanced heart failure. In $71 \%$ of cases, they were in WHO class III or IV, similarly to the REVEAL FCC (73\%) [26] and the French registry (75\%) [12]. This number is even more dramatic as it is similar to the one reported on the 20-year-old NIH cohort (71\%) [21]. The combination of high RAP with an advanced functional class on presentation signalizes that more effort is needed for early identification and referral of patients to expert centers, as there is evidence that treating patients in WHO class II has a positive impact on patients' outcomes [28].

CTEPH patients had similar haemodynamics compared to PAH, except for mean RAP, which was significantly higher. This finding that was not reproduced in the Swiss cohort [22], is higher than that reported in the BENEFIT study [23] and in the Cambridge cohort [29]. Higher right ventricular filling pressures were accompanied by a higher number of patients being referred to WHO class III or IV (78\%), although similar to the proportion reported in the literature [29]. This may indicate late identification and the referral of these potentially curable patients.

Although having high WHO functional class at presentation, most PAH patients were not treated with diuretics when referred to the expert centers. The same was true regarding CTEPH patients; however, there was a trend for more intense anticongestive medication in this group. Our results are comparable to those from the Swiss registry [22]. Late referral to specialized centers may be in part due to the lack of an nationwide reference network and availability of oral vasodilator drugs for PAH treatment, as less specialized centers may delay transfer patients to expert centers [12]. In our population, no patient was treated with specific pulmonary vasodilators before being referred to the specialized centers.

Vasoreactivity testing was held in 29 of $46 \mathrm{PAH}$ patients (63\%) and was positive in 6 patients (21\%). This value is significantly higher than that reported by the French cohort (10.3\%) [12] but similar to the Swiss registry (20\%) [17], although the latter included 8 CTEPH patients. Selection bias, differences in the definition of acute responders and in the treatment protocols used nationwide, may be responsible for the inconsistencies. Publication of national guidelines may help to standardize the care for this group of patients.

The progress in prognosis is inseparable from the advances in pulmonary vasodilator therapy. There are three classes of selective vasodilator drugs that target three critical pathways in PAH (prostacyclin, nitric oxide, and endothelin1) [2], all being available in Portugal. All of them have their efficacy demonstrated in several randomized controlled trials regarding functional capacity, exercise tolerance, haemodynamics, and other endpoints [30]. Moreover, a recent metaanalysis confirmed the impact of pulmonary vasodilators on short-term survival [11]. Overall, in our cohort the one-year survival for PAH patients was $93.5 \%$, similar to the REVEAL (91.0\%) [20] and the Swiss cohort (89.0\%) [22] but higher than the French cohort (85.7\%) [12]. The differences may be accounted by the small number of events in our cohort ( 5 deaths) and the relative higher proportion of patients with CHD-associated PAH (21.7\%) compared with the REVEAL (11.8\%), Swiss (approximately 14.3\%), and French cohorts $(0 \%)[12,17,20]$. These patients clearly have a better prognosis [31], and thus they may have contributed to the positive survival results.

CTEPH patients had a similar one-year survival (93.9\%); only five patients underwent PEA, a potentially curative procedure that probably had impact on prognosis, as no patient died on follow-up. All CTEPH patients are assessed by the local medical $\mathrm{PH}$ team, and the potential surgical candidates are discussed directly with the foreign referral center, as the procedure was not routinely performed in our country. Not operated patients had a survival of $92.9 \%$. For these patients and for those with residual $\mathrm{PH}$ after PEA, pulmonary vasodilator drug therapy is a class IIb C recommendation in the ESC guidelines [9]. In our cohort, two thirds of CTEPH patients started specific therapy, a number that is comparable to other series [29]. Interestingly, the one-year survival of the not operated CTEPH patients was similar to that recently reported in the literature (96\%) [32].

Our estimated PAH annual incidence between 1.5 and 2.2 cases per million inhabitants is in line with the published incidences in other countries: Belgium (1.7 per million) [33], Israel (1.4 per million) [34], France (2.4 per million) [12], Switzerland (2.4 per million) [17], and USA (2.0 per million) 
[26]. The wide range between the most conservative estimate and the higher value is also observed in other series, as in France, where there are very high regional differences in PAH prevalence, ranging from 5 to 25 per million inhabitants per year [12]. CTEPH had, in our population, an estimated incidence of 1.1 cases per million, a number similar to that reported in the United Kingdom in 2001 (1.02 cases per million) but lower than that in 2005 (1.75 cases per million) [29].

Our study has several limitations. First, although we included data from five expert PH centers in Portugal, there are patients followed in other hospitals across the country. This had impact on incidence calculations, namely, an underestimation of values, both in PAH and CTEPH. Second, we used strict inclusion and exclusion criteria for the recruitment of patients in this registry to ensure a homogenous population. This has caused the exclusion of all patients without a RHC on the databases, whether or not there was one available on the clinical files. However, as our mean hemodynamic values, demographics, and functional class data are in line with those published on the literature, we believe that our population is representative and has external validity. Thirdly, we decided not to include groups 2, 3, and 5 patients, as there were significant differences among centers regarding the clinical characteristics of these patients. A careful analysis was made, but avoiding confounding factors that are frequent in observational studies may have been impossible [35].

In conclusion, the present unique study reports for the first time data on the epidemiology, clinical characteristics, and prognosis of PAH and CTEPH patients in Portugal. We conclude that overall PAH incidence is similar to that reported in other European series, but patients are still being diagnosed late in the course of their disease. We also report that CHD-PAH is an important etiology in our country and may need special attention. The one-year survival analysis of our incident cohort exceeds $93 \%$, a value that reflects access to contemporary treatment of PAH, being a strong incentive to the continuous work being developed by all the community members involved in this disease. We demonstrated that a combined and organized registry is possible and is a useful tool to obtain quality data for clinical decision-making that compares well with data from other registries. Our findings encourage the amplification and maintenance of a nationwide registry by the combined effort of all the physicians caring these patients, aiming for a better care and prognosis of $\mathrm{PH}$ patients.

\section{Conflict of Interests}

Abilio Reis is the coproprietary of the dedicated pulmonary hypertension software PAHTool. The remaining authors have no conflict of interests to disclose.

\section{Acknowledgment}

The authors thank Actelion Portugal Lda. for supporting the development of the dedicated software (PAHTool) and data entry.

\section{References}

[1] G. E. D’Alonzo, R. J. Barst, S. M. Ayres et al., "Survival in patients with primary pulmonary hypertension," Annals of Internal Medicine, vol. 115, no. 5, pp. 343-349, 1991.

[2] M. Humbert, O. Sitbon, and G. Simonneau, "Treatment of pulmonary arterial hypertension," The New England Journal of Medicine, vol. 351, no. 14, pp. 1425-1473, 2004.

[3] M. Humbert, G. Simonneau, and L. J. Rubin, "A decade of achievement in pulmonary hypertension," European Respiratory Review, vol. 20, no. 122, pp. 215-217, 2011.

[4] M. Humbert, O. Sitbon, A. Chaouat et al., "Survival in patients with idiopathic, familial, and anorexigen-associated pulmonary arterial hypertension in the modern management era," Circulation, vol. 122, no. 2, pp. 156-163, 2010.

[5] V. V. McLaughlin, "Looking to the future: a new decade of pulmonary arterial hypertension therapy," European Respiratory Review, vol. 20, no. 122, pp. 262-269, 2011.

[6] S. Hatano and T. Strasser, "Primary pulmonary hypertension?" Report on A WHO Meeting, World Health Organization, Geneva, Switzerland, 1973.

[7] G. Simonneau, I. M. Robbins, M. Beghetti et al., "Updated clinical classification of pulmonary hypertension," Journal of the American College of Cardiology, vol. 54, no. 1, pp. S43-S54, 2009.

[8] A. Reis, N. Rocha, R. Barros et al., "Guidelines for the management of pulmonary hypertension patients," Revista Portuguesa de Cardiologia, vol. 17, pp. S7-S85, 2010.

[9] N. Galiè, M. M. Hoeper, M. Humbert et al., "Guidelines for the diagnosis and treatment of pulmonary hypertension: the Task Force for the Diagnosis and Treatment of Pulmonary Hypertension of the European Society of Cardiology (ESC) and the European Respiratory Society (ERS), endorsed by the Internat," European Respiratory Journal, vol. 30, no. 6, pp. 2493 2537, 2009.

[10] A. M. Keogh, E. Mayer, R. L. Benza et al., "Interventional and surgical modalities of treatment in pulmonary hypertension," Journal of the American College of Cardiology, vol. 54, supplement 1, pp. S67-S77, 2009.

[11] N. Galiè, A. Manes, L. Negro, M. Palazzini, M. L. BacchiReggiani, and A. Branzi, "A meta-analysis of randomized controlled trials in pulmonary arterial hypertension," European Heart Journal, vol. 30, no. 4, pp. 394-403, 2009.

[12] M. Humbert, O. Sitbon, A. Chaouat et al., "Pulmonary arterial hypertension in France: results from a national registry," The American Journal of Respiratory and Critical Care Medicine, vol. 173, no. 9, pp. 1023-1030, 2006.

[13] Z.-C. Jing, X.-Q. Xu, Z.-Y. Han et al., "Registry and survival study in Chinese patients with idiopathic and familial pulmonary arterial hypertension," Chest, vol. 132, no. 2, pp. 373379, 2007.

[14] A. J. Peacock, N. F. Murphy, J. J. V. McMurrey, L. Caballero, and S. Stewart, "An epidemiological study of pulmonary arterial hypertension," European Respiratory Journal, vol. 30, no. 1, pp. 104-109, 2007.

[15] T. Thenappan, S. J. Shah, S. Rich, and M. Gomberg-Maitland, "A USA-based registry for pulmonary arterial hypertension: 19822006," European Respiratory Journal, vol. 30, no. 6, pp. 1103-1110, 2007.

[16] M. D. McGoon, A. Krichman, H. W. Farber et al., "Design of the REVEAL registry for US patients with pulmonary arterial hypertension," Mayo Clinic Proceedings, vol. 83, no. 8, pp. 923931, 2008. 
[17] C. Tueller, H. Stricker, P. Soccal et al., "Epidemiology of pulmonary hypertension: new data from the swiss registry," Swiss Medical Weekly, vol. 138, no. 25-26, pp. 379-384, 2008.

[18] M. Gomberg-Maitland and E. D. Michelakis, "A global pulmonary arterial hypertension registry: is it needed? Is it feasible?" Chest, vol. 137, supplement, no. 6, pp. 95S-101S, 2010.

[19] N. Galiè, M. M. Hoeper, M. Humbert et al., "Guidelines for the diagnosis and treatment of pulmonary hypertension," European Respiratory Journal, vol. 34, no. 6, pp. 1219-1263, 2009.

[20] R. L. Benza, D. P. Miller, M. Gomberg-Maitland et al., "Predicting survival in pulmonary arterial hypertension: insights from the registry to evaluate early and long-term pulmonary arterial hypertension disease management (REVEAL)," Circulation, vol. 122, no. 2, pp. 164-172, 2010.

[21] S. Rich, D. R. Dantzker, S. M. Ayres et al., "Primary pulmonary hypertension: a national prospective study," Annals of Internal Medicine, vol. 107, pp. 216-223, 1987.

[22] M. Fischler, R. Speich, L. Dorschner et al., "Pulmonary hypertension in Switzerland: treatment and clinical course," Swiss Medical Weekly, vol. 138, no. 25-26, pp. 371-378, 2008.

[23] X. Jaïs, A. M. D’Armini, P. Jansa et al., "Bosentan for treatment of inoperable chronic thromboembolic pulmonary hypertension chronic thromboembolic pulmonary hypertension ", Journal of the American College of Cardiology, vol. 52, no. 25, pp. 2127-2134, 2008.

[24] V. Pengo, A. W. A. Lensing, M. H. Prins et al., "Incidence of chronic thromboembolic pulmonary hypertension after pulmonary embolism," The New England Journal of Medicine, vol. 350, no. 22, pp. 2257-2323, 2004.

[25] R. Souza, M. Humbert, B. Sztrymf et al., "Pulmonary arterial hypertension associated with fenfluramine exposure: report of 109 cases," European Respiratory Journal, vol. 31, no. 4, pp. 343$348,2008$.

[26] A. E. Frost, D. B. Badesch, R. J. Barst et al., "The changing picture of patients with pulmonary arterial hypertension in the United States: how REVEAL differs from historic and non-US contemporary registries," Chest, vol. 139, no. 1, pp. 128-137, 2011.

[27] E. Hachulla, V. Gressin, L. Guillevin et al., "Early detection of pulmonary arterial hypertension in systemic sclerosis: a French nationwide prospective multicenter study," Arthritis and Rheumatism, vol. 52, no. 12, pp. 3792-3800, 2005.

[28] N. Galiè, L. Rubin, M. Hoeper et al., "Treatment of patients with mildly symptomatic pulmonary arterial hypertension with bosentan (EARLY study): a double-blind, randomised controlled trial," The Lancet, vol. 371, no. 9630, pp. 2093-2100, 2008.

[29] R. Condliffe, D. G. Kiely, J. S. R. Gibbs et al., "Improved outcomes in medically and surgically treated chronic thromboembolic pulmonary hypertension," The American Journal of Respiratory and Critical Care Medicine, vol. 177, no. 10, pp. 11221127, 2008.

[30] H. A. Ghofrani, O. Distler, F. Gerhardt et al., "Treatment of pulmonary arterial hypertension (PAH): updated recommendations of the Cologne Consensus Conference 2011," International Journal of Cardiology, vol. 154, supplement 1, pp. S20-S33, 2011.

[31] W. E. Hopkins, L. L. Ochoa, G. W. Richardson, and E. P. Trulock, "Comparison of the hemodynamics and survival of adults with severe primary pulmonary hypertension or Eisenmenger syndrome," Journal of Heart and Lung Transplantation, vol. 15, no. 1, pp. 100-105, 1996.
[32] R. J. Hughes, X. Jais, D. Bonderman et al., "The efficacy of bosentan in inoperable chronic thromboembolic pulmonary hypertension: a 1-year follow-up study," European Respiratory Journal, vol. 28, no. 1, pp. 138-143, 2006.

[33] L. Abenhaim, Y. Moride, F. Brenot et al., "Appetite-suppressant drugs and the risk of primary pulmonary hypertension. International Primary Pulmonary Hypertension Study Group," The New England Journal of Medicine, vol. 335, pp. 609-616, 1996.

[34] L. Appelbaum, M. Yigla, D. Bendayan et al., "Primary pulmonary hypertension in Israel: A national survey," Chest, vol. 119, no. 6, pp. 1801-1806, 2001.

[35] V. V. McLaughlin and S. Suissa, "Prognosis of pulmonary arterial hypertension: the power of clinical registries of rare diseases," Circulation, vol. 122, no. 2, pp. 106-108, 2010. 


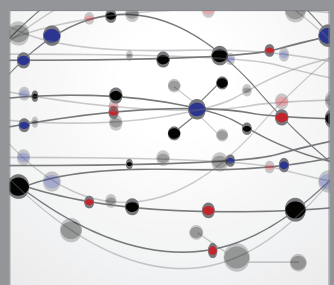

The Scientific World Journal
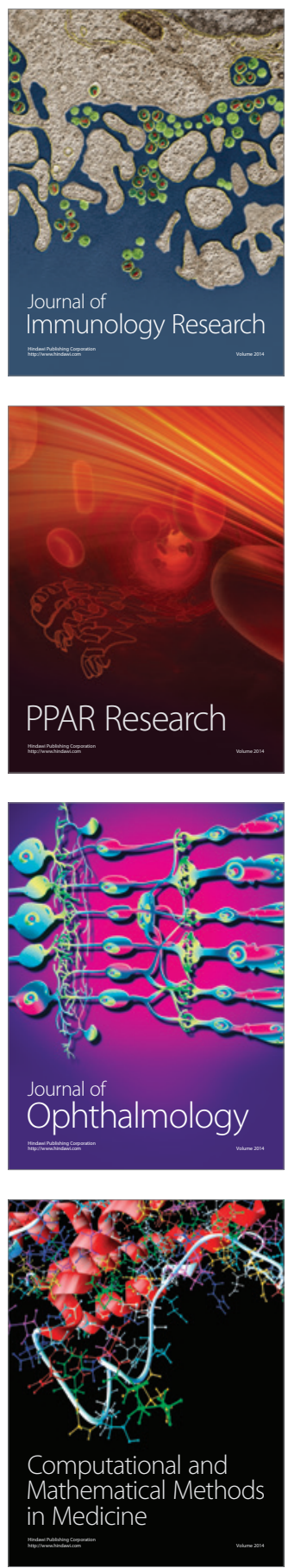

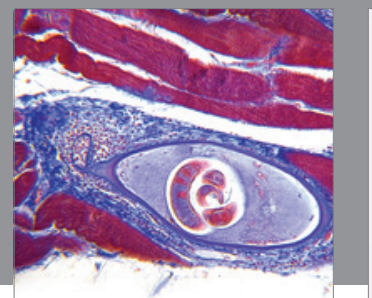

Gastroenterology

Research and Practice
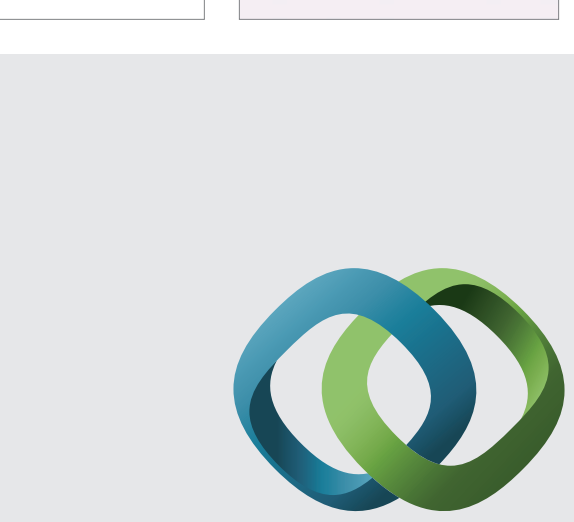

\section{Hindawi}

Submit your manuscripts at

http://www.hindawi.com
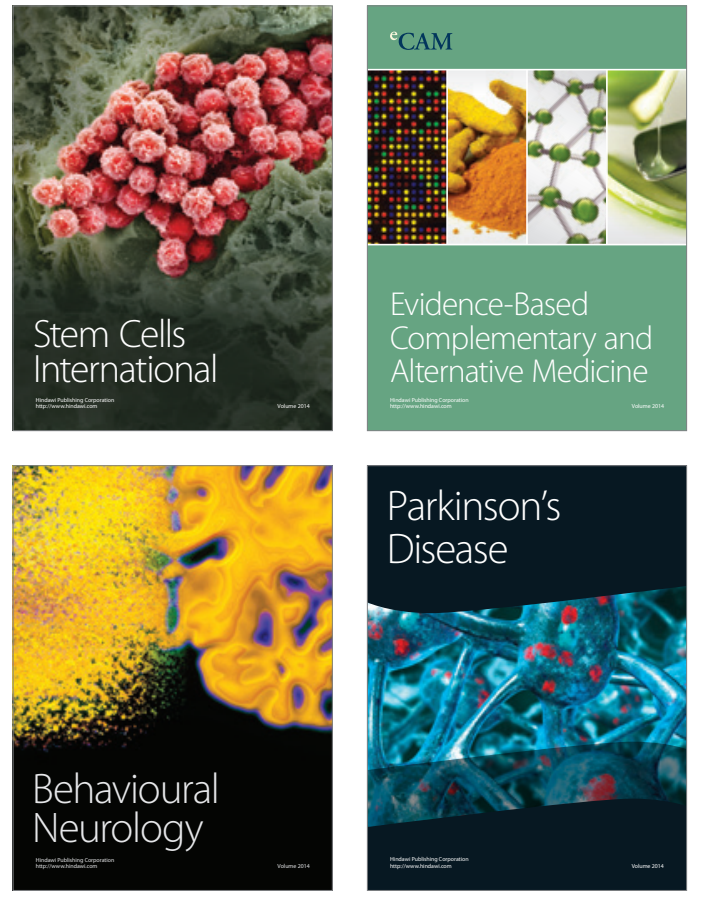
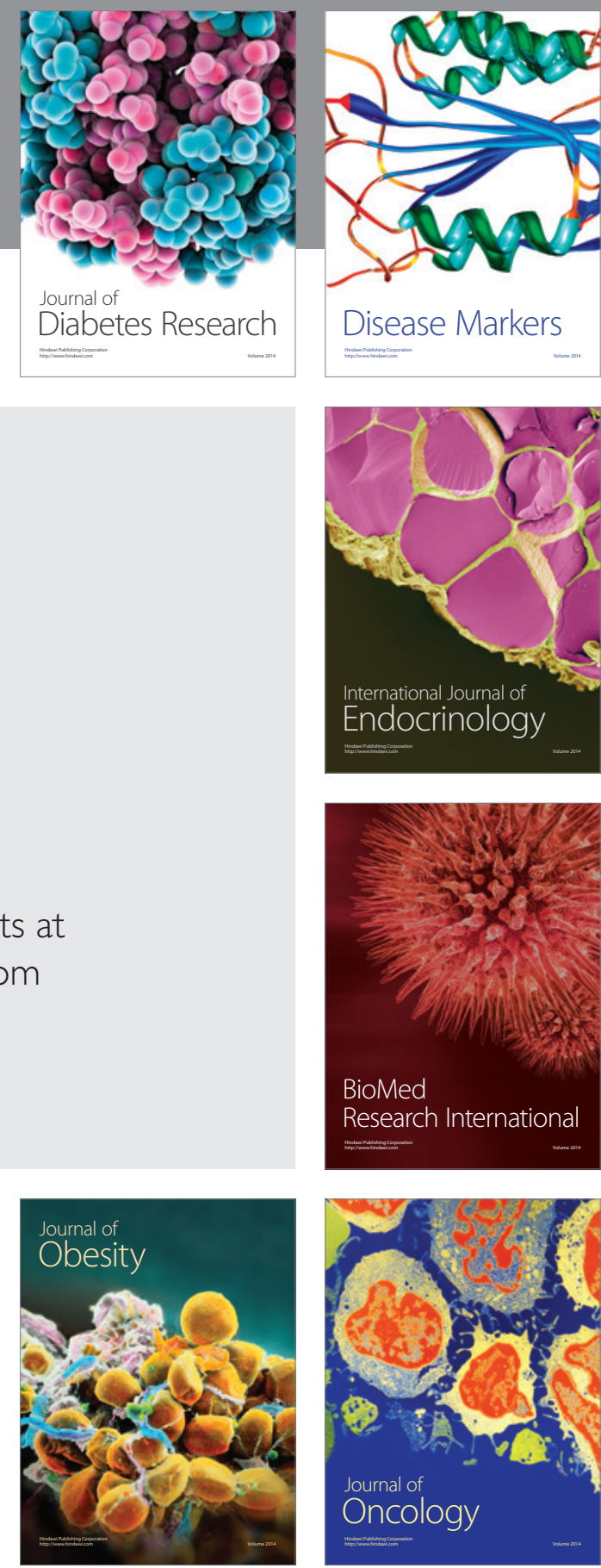

Disease Markers
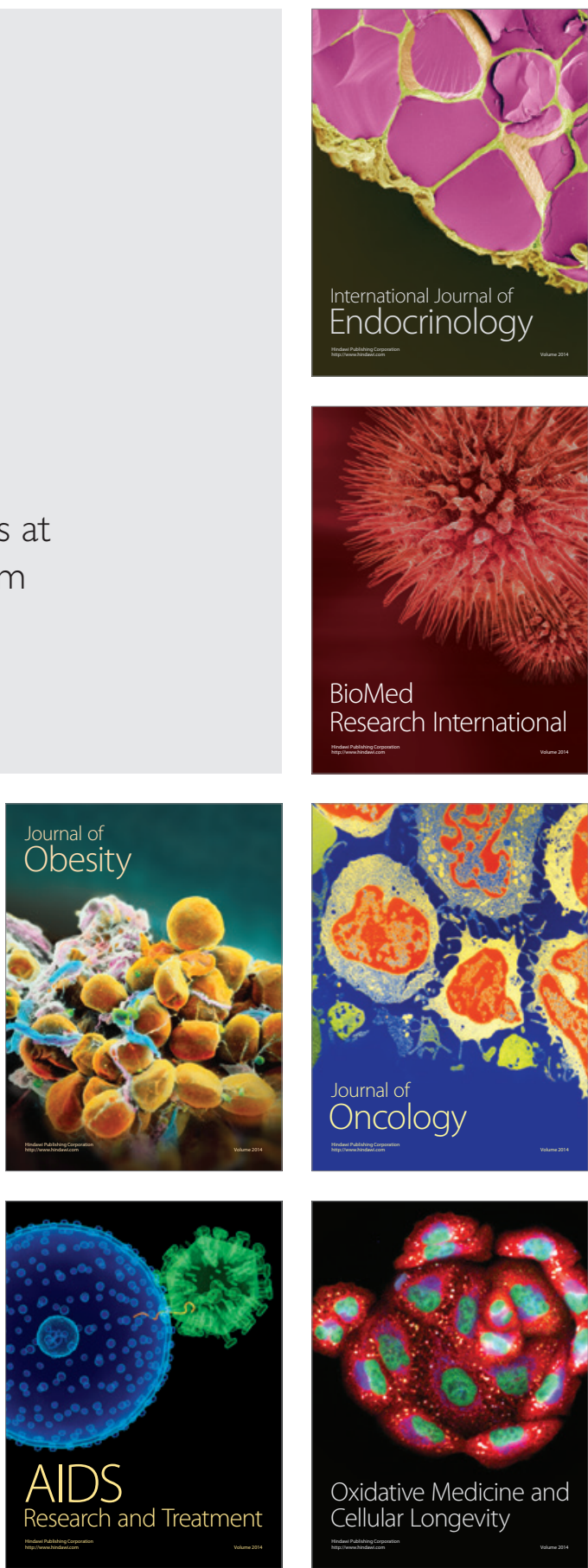